\title{
Fisiomotricidade e limiares de dor: efeitos de um programa de exercícios na autonomia funcional de idosas osteoporóticas
}

\section{Fisiomotricity and threshold of pain: effects of a physical exercise program in the functional autonomy of osteoporotic elderly woman}

\author{
Karla Virgínia Bezerra de Castro ${ }^{[a]}$, André Luiz dos Santos Silva ${ }^{[b]}$, \\ Jacqueline Maria Maranhão Pinto Lima ${ }^{[\mathrm{c}]}$, Walter Jacinto Nunes ${ }^{[\mathrm{d}]}$, \\ Maurício Rocha Calomeni ${ }^{[e]}$, Vernon Furtado da Silva ${ }^{[f]}$
}

[a] Mestranda em Ciência da Motricidade Humana pela Universidade Castelo Branco, RJ, Centro de Ensino Universitário do Maranhão, São Luis, MA - Brasil, homepage: www.ceuma.br

[b] Doutor em Ciências Médicas, Coordenador do Laboratório de Aprendizagem Neural e Performance Motora (LANPEM), Novo Hamburgo, RS - Brasil, homepage: http://www.feevale.br/home

[c] Mestranda em Ciência da Motricidade Humana pela Universidade Castelo Branco, RJ, Centro Universitário do Maranhão, São Luis, MA - Brasil, e-mail: ceuma@elo.com.br

[d] Doutor em Análise de Ensino em Ciência da Motricidade Humana, Universidade Federal Rural do Rio de Janeiro (UFRRJ), Seropedica, RJ - Brasil, e-mail: nunes.walter@ig.com.br

[e] Mestre em Ciência da Motricidade Humana, Pesquisador do Laboratório de Aprendizagem Neural e Performance Motora (LANPEM-UCB/RJ),São Fídélis, RJ - Brasil, e-mail: mauriciocalomeni@gmail.com

[f] Pós-Doutorado em Sistemas Dinâmicos do Movimento, Coordenador do Laboratório de Aprendizagem Neural e Performance Motora (LANPEM-UCB/RJ), Rio de Janeiro, RJ - Brasil, e-mail: vernonfurtado2005@yahoo.com.br

\section{Resumo}

Introdução: A atividade física é importante para a manutenção da autonomia funcional do homem e crucial em idosos. Todavia, alguns fatores, como a dor, limitam a participação deles em programas de atividade física. Objetivo: Investigar se, em função de um programa de atividades físicas estruturado de maneira a limitar a dor, poder-se-ia garantir a aderência de idosas sofrendo de osteoporose/osteopenia e, dessa forma, promover melhoras na autonomia funcional destas. Desenvolvimento: A amostra foi composta por 30 mulheres, entre 65 e 70 anos, divididas em dois grupos definidos em função de um diagnóstico de dor. Materiais e métodos: Aplicou-se um programa definido pelo termo fisiomotriz, de baixo impacto, caracterizado por lentidão nas mudanças articulares, progredindo em quatro níveis que foram distribuídos em 48 encontros. Os dados da dor e da autonomia funcional, obtidos por meio do teste de dor em escala analógica visual e do protocolo de GDLAM foram estudados por estatística descritiva e inferencial, com o índice ALFA $\pm 0,05$. O instrumento foi uma Análise de Variância de Kruskal Wallis com índice Qui-quadrado $\left(\mathrm{Chi}^{2}\right)$. Resultados: Os resultados revelaram melhora da autonomia funcional e 
diminuição de dor relatada pelas participantes, fato que consequentemente implementou a aderência destas até ao final do programa. Conclusão: Conclui-se que o programa fisiomotriz, aplicado em idosas com osteopenia e/ou osteoporose, foi uma forma eficaz na promoção da melhora de sua autonomia funcional, que pode ser atribuída, pelo menos em parte, à diminuição de dor antes relatada pelas idosas.

Palavras-chave: Dor. Autonomia funcional. Exercício e envelhecimento. Idoso.

\section{Abstract}

Introduction: Physical activity that is important for people functional autonomy at old ages is crucial. Some factors limit elderly people in this kind of programs. Pain is one of them. This study examined whether a physical activity program, structured for limiting pain feeling would ensure the adderence of an elderly group suffering from osteoporosis/ osteopenia, and therefore, promote improvements in their functional autonomy. Development: Thirty women with the above specified problem, 65 to 70 years old, divided into two groups defined on the basis of a pain diagnosis. Methods: An exercises program previously defined by the fisiomotriz term was applied, being of low impact, characterized by slow changes in the joints, progressing to four difficult levels, distributed in 48 meetings. Data from the pain and functional autonomy, measured by the pain visual analogue scale and the GDLAM protocol were statistically studied and the index ALFA fixed was \pm 0.05 . The analisys of variance was the Kruskal Wallis, with the chi-square index of independence. Results: The results revealed improved functional independence and decrease in pain, as reported by the participants with osteopenia/osteoporosis. This fact consequently implemented the adhesion of these by the end of the program. Conclusion: It was conclude the program fisiomotriz applied in elderly women with osteopenia and/or osteoporosis was an effective way in promoting the improvement of the functional autonomy of the elderly woman studied. This improvement can be attributed, at least in part, to decrease in pain as compared to levels reported priorly to the program initiation.

Keywords: Pain. Functional autonomy. Ageing and exercise. Elderly.

\section{Introdução}

$\mathrm{Na}$ maioria dos casos de doenças incapacitantes, a dor é condição consequente, variando em intensidade, de acordo com o nível da doença. Porém, não necessariamente este mal está intrinsecamente associado a uma condição doentia. Pesquisadores verificaram e enfatizaram que a alta prevalência de dor na população idosa está normalmente associada a desordens crônicas, destacando-se as artrites e osteoporoses, tendo influência dos altos níveis de inabilidade funcional e na maior fragilidade corporal, relacionando-se à perda da autonomia funcional (1-4). Embora seja uma condição que qualquer ser humano procura evitar, independentemente de suas conceituações, percebe-se que a dor constitui-se em uma experiência privada, totalmente subjetiva, não resultando apenas de características teciduais, abrangendo, também, fatores de várias ordens, incluindo as emocionais, culturais e individuais (5), sendo, paradoxalmente, um elemento crucial para a proteção e manutenção da vida $(6,7)$. Vários estudos $(8,9)$, dentre eles os de Lin et al. (10), relatam que a utilização de exercícios isométricos, ativos livres e contrarresistidos, assim como o relaxamento de estruturas tensas ou contraturadas, são capazes de reduzir edemas e processos inflamatórios, melhorando as condições circulatórias e favorecendo o alívio da dor e minimização da incapacidade funcional, pois movimentos aplicados sob critérios 
personalizados podem auxiliar a dessensibilização de áreas dolorosas por meio da estimulação exteroceptiva, contanto que sejam adequadas à capacidade de cada indivíduo.

Nesse contexto, Silva e Lage (11) citam que práticas as quais utilizam posturas estáveis e confortáveis, respiração diafragmática com expirações lentas, sem esforço excessivo durante as contrações musculares podem favorecer a inibição de atividade cerebral de áreas relacionadas à dor. Indivíduos osteoporóticos, quando submetidos a programas de atividade física, apresentaram melhora na sensação de dor e diminuição significativa no uso de analgésicos, com consequente aumento na mobilidade e na capacidade funcional, além de ganho de massa óssea (12). Já o sedentarismo, a incapacidade funcional e a dependência se destacam como os grandes vilões causadores das maiores adversidades, principalmente no processo do envelhecimento e na perda de massa óssea $(13,14)$. Os benefícios da atividade física sobre os vários fatores que estruturam a saúde e a competência social do homem em grande parte estão relacionados à autonomia funcional de idosos, decorrentes de exercícios de força muscular, flexibilidade e capacidade aeróbica (15-21). Outras têm demonstrado que exercícios com carga conseguem minimizar a perda progressiva de densidade óssea e, ainda, melhorar a qualidade de vida em indivíduos idosos (12-14).

Entretanto, para que surta um efeito positivo, a adesão ao tratamento e/ou à atividade física precisa ser completa, obedecendo critérios de influência à adesão e à pratica regular de uma terapia física. Entre eles está inserida a crença no tratamento, que na maioria das vezes encontra-se comprometida em virtude de experiências anteriores insatisfatórias (22). De acordo com o posicionamento oficial da Sociedade Brasileira de Medicina do Esporte e da Sociedade Brasileira de Geriatria e Gerontologia, um programa de atividade física para a população idosa, deve incluir condicionamento cardiorrespiratório, endurance e força muscular, priorizando a maximização do contato social, reduzindo a ansiedade e a depressão que muitas vezes acometem o idoso.

Em pacientes com dores crônicas, a adesão às terapêuticas oferecidas é bastante baixa, abrindo uma lacuna para a apresentação de intervenções que aperfeiçoem a adesão (22). Como a presença de dor pode ser um motivo potencial para um grande contingente de idosos não participar de programas de atividades físicas (23), a atenuação efetiva deste mal se constitui necessidade de ordem imperativa a uma solução.

O objetivo deste estudo foi investigar se em função da minimização do fator dor e da aderência ao programa de exercícios, mulheres idosas com osteoporose/osteopenia poderiam melhorar em termos de autonomia funcional.

\section{Procedimentos}

\section{Tipologia do estudo}

Tratou-se de um estudo de natureza ex-postfact, considerando-se que os membros da amostra já eram portadores dos fatores causadores de dor no momento da intervenção metodizada. A verificação da eficácia do programa de exercícios ocorreu dentro de um procedimento de campo controlado, sendo esta realizada em uma clínica especializada em tratamento de idosos, localizada na cidade de São Luís do Maranhão.

\section{Amostra}

A população de referência para este estudo foi definida em relação a mulheres senescentes com perda de massa óssea, dor crônica e limitação funcional. Nela, buscou-se, sob forma de conveniência, um grupo amostral que constituiu a base para as operacionalizações experimentais da pesquisa em si. Todas estavam vinculadas ao setor de fisioterapia da clínica vindas de várias partes e bairros da cidade de São Luís, Maranhão. 
Os critérios que caracterizavam a inclusão de indivíduos na pesquisa em pauta foram: ter entre 65 e 70 anos de idade e ser portadora de osteoporose e/ou osteopenia em sítios corporais específicos (colo do fêmur, coluna lombar). Para isso, foram considerados os seguintes termos aceitos pela OMS: osteopenia, quando a perda é de 1 a 2,5 desvios-padrão (DP) identificados pelo exame; osteoporose, quando a perda é maior do que 2,5 desvios-padrão (DP). Foram também inseridas na amostra idosas queixosas de dor e com limitação funcional para a realização de suas AVD's, assim como as que apresentavam a impossibilidade de realizar atividades físicas convencionais, com encaminhamento médico para fisioterapia e que tivessem em mãos o exame de densitometria óssea do ano vigente para a comprovação do diagnóstico anteriormente referido. Também foi critério de inclusão estar fazendo uso de cálcio e apresentar habilidade psicomotora para a compreensão e realização dos exercícios, bem como a disposição e vontade para a participação efetiva. Assim sendo, foram excluídas do estudo pacientes que (1) apresentaram qualquer patologia que as impossibilitasse de realizar, na íntegra, os exercícios do programa ou que estes pudessem pôr em risco o quadro preexistente de problemas de saúde ao longo da intervenção; (2) as que não tiveram frequência igual ou superior a 80\% no processo de intervenção; (3) participantes de qualquer outro tipo de atividade física que não fosse a do programa; (4) pacientes que não se encontrassem sedentárias há, no mínimo, seis meses (5) ou que possuíssem desvio-padrão (DP) abaixo de 1.0, identificado pelo exame de densitometria óssea. Também não foram incluídas (6) pacientes que faziam uso de analgésico ou terapia de reposição hormonal.

Considerando-se os itens de inclusão e exclusão da população anteriormente descrita, foram finalmente selecionadas 29 delas, compondo-se, assim, o grupo amostral.

\section{Ética da pesquisa}

Este estudo foi desenvolvido respeitando as normas estabelecidas na Resolução n. 196/96 do Conselho Nacional de Saúde, de 10/10/1996, com relação à realização de pesquisa em seres humanos, sob protocolo n. 0075/2008 UCB/VREPGPE/COMEP/PROCIMH.

Todos os participantes da amostra do estudo, assim como a instituição na qual se realizou a pesquisa, receberam um termo de consentimento livre e esclarecido para ser lido e devidamente assinado em caso de aceitação. Neste termo constaram os aspectos relativos ao estudo como objetivo, caráter de voluntariedade à participação, liberdade para aderir e/ou sair do estudo sob conveniência própria, benefícios e possíveis riscos inerentes à participação, procedimentos de avaliação e procedimentos de emergência, entre outros.

\section{Procedimentos}

A pesquisa foi desenvolvida em três etapas avaliativas, cada uma com suas especificidades, a saber:

\section{Primeira etapa: avaliação de contexto}

Nesta avaliação foi realizada uma sessão de pré-testes com o propósito de homogeneização da amostra, executado da seguinte maneira:

a) Entrevista para apresentação e explicação do estudo e assinatura do termo de consentimento;

b) Aplicação de um questionário socioeconômico;

c) Aplicação do teste de dor por escala analógica visual de onze pontos, que consiste em uma linha reta, de $10 \mathrm{~cm}$, que representa o contínuo dor, ancorada pelas palavras sem dor e pior dor. Foi solicitado que a participante marcasse na linha o lugar que representava a intensidade da dor sentida naquele momento. O observador mediu, em centímetros, a 
distância entre a extremidade ancorada pelas palavras sem dor e a marca feita pelo paciente, que correspondeu à intensidade de sua dor;

d) Aplicação do protocolo GDLAM, visando à avaliação da autonomia funcional do grupo. Conhecido como um protocolo para estudo do desenvolvimento Latino-Americano para a Maturidade-GDLAM, inclui tarefas tais como caminhar por $10 \mathrm{~m}$ (C10m), levantar-se da posição sentada (LPS), da posição decúbito ventral (LPDV), da cadeira e locomoverse pela casa (LCLC) e o teste de vestir e tirar uma camiseta (VTC). Todos os itens são utilizados para calcular o índice GDLAM-IG, obtido por meio de normalização entre os 5 testes de autonomia, estimando os valores de classificação pela fórmula:

$$
\mathrm{IG}=[(\mathrm{C} 10 \mathrm{~m}+\mathrm{LPS}+\mathrm{LPDV}+\mathrm{VTC}) \mathrm{x} 2]+\mathrm{LCLC}
$$

4

e) registro dos valores da densitometria óssea do ano vigente para a avaliação da massa óssea, todos realizados no serviço de densitometria óssea (SDO), acompanhados com o respectivo laudo médico.

\section{Segunda etapa: intervenção}

Nesta etapa a amostra foi dividida em dois grupos, um com 15 indivíduos e o outro, por causa dos critérios de exclusão, com 14 indivíduos, respeitando os critérios de mais dor - valores acima de 5 na escala analógica visual - e menos dor - valores abaixo de 5 na escala analógica visual. A seguir foi aplicado um programa de exercícios de baixo impacto e graduado em intensidade, sendo esta adequada ao nível de dor de cada participante. Este programa, definido pelo termo "fisiomotriz", foi desenvolvido de forma idêntica para os dois grupos, durante dezesseis semanas, com atendimentos três vezes por semana em sessões de 50 minutos. O programa de natureza fisiomotriz caracteriza-se pela utilização de exercícios cujas formulações são baseadas em princípios biomecânicos apropriados, garantindo-se neles condições de flutuabilidade articular e muscular com o mínimo de atrito entre articulações segmentares e intersegmentares, mantendo-se níveis lentos de execução, buscando, com isso, o máximo de minimização da dor, de acordo com os pressupostos da Teoria de Melzack e Wall (24) em referência à permeabilidade de estímulos via portais de dor. Outra característica fundamental da execução dos movimentos refere-se à constância da mentalização sobre os movimentos em execução, garantindo controle executivo apurado na relação corpo e mente. Suas bases foram estabelecidas em longo período de estudos e trabalhos práticos, desenvolvidos pelo Laboratório de Aprendizagem Neural e Performance Motora (LANPEM/UCB/RJ/CNPq) que tem investigado, nos últimos anos, os efeitos do programa fisiomotriz sobre diferentes aspectos e tendo resultados extremamente positivos e promissores, os quais têm sido apresentados em periódicos científicos, congressos e dissertações de mestrado (25-29).

Os exercícios que compuseram o Nível I ( $1^{\mathrm{a}}$ a $5^{\mathrm{a}}$ sessão) constituíram-se a prática da consciência corporal, associadamente à compreensão de padrões respiratórios fisiologicamente adequados. O Nível II ( $6^{\mathrm{a}}$ a $15^{\mathrm{a}}$ sessão) foi composto por exercícios leves, com movimentos de mínima amplitude, obedecendo-se aos pressupostos inerentes à teoria de Melzack e Wall (24) com sutil contração isométrica local. No nível III (16 a $30^{\mathrm{a}}$ sessões), exercícios com contrações leves, envolvendo movimentos de pequena amplitude, seguidas por contração isométrica global (leve) associada à contração isotônica livre (sem carga). Já o nível IV $\left(30^{\mathrm{a}}\right.$ a $48^{\mathrm{a}}$ sessão) foi formado por movimentos de grande amplitude com contração isométrica global máxima e/ou isotônica com carga, seguindo-se uma evolução no nível de complexidade de execução. Desta forma, o protocolo de exercícios iniciou-se com movimentos de pequena amplitude articular e leve contração isométrica local evoluindo para global, sempre associado a um padrão expiratório. 
A progressão ou evolução se deu pelo aumento de amplitude de movimento e aumento de tensão e tempo durante as contrações isométricas, quando passaram a ser adicionadas forças externas (cargas), evolução postural (deitada, sentada, em pé), complexidade de movimentos (olhos abertos, depois fechados), apoio bi-podal seguido de unipodal, velocidade gradativamente mais lenta, e de exercícios para dupla.

\section{Terceira etapa: avaliação da intervenção}

Após 16 semanas, isto é, 48 atendimentos da referida intervenção, foi realizado um pós-teste para se obter os dados finais relativos aos efeitos da intervenção por si, momento que se fez uso dos mesmos instrumentos empregados no pré-teste.

Além do questionário e das escalas mencionadas anteriormente, também foram utilizados neste trabalho os seguintes instrumentos:
a) Um cronômetro (Cássio, Malaysia);
b) Uma trena (Sanny, Brasil);
c) Um colchonete e uma cadeira com $50 \mathrm{~cm}$ de altura do assento ao solo;
d) Uma camiseta tamanho G.

\section{Tratamento estatístico}

Os dados foram analisados pelo programa SPSS for Windows 16.0, a estatística inferencial foi a Análise de Variância de Kruskal Wallis com o índice Qui-Quadrado de independência (Chi ${ }^{2}$. Em todos os testes, o nível de significância definido para o teste da hipótese do estudo foi de ALFA $\pm 5 \%$. Utilizouse, também, instrumento da estatística descritiva, visando-se informações que mediassem melhor o entendimento dos dados resultantes, em referência aos efeitos da manipulação das variáveis do estudo.

\section{Resultados}

A título de facilitar o entendimento sobre os resultados, ora em fase de discussão, convém lembrar que se referem à comparação da eficácia de um método de exercícios com base fisioterapêuticas aplicado a um grupo composto por 15 idosas entre 65 e 70 anos, todas manifestando dor abaixo do nível 5.0 da Escala Analógica Visual e, a um outro, composto por 14 idosas, na mesma faixa etária, com dor acima de 5.0 pontos verificada na mesma Escala. Conforme se pode observar na Tabela 1, os resultados demonstram a ocorrência de reduções nos índices de dor (ID) em todas as idosas, após participação no programa fisiomotriz aplicado em benefício da diminuição de dor.

Tabela 1 - Distribuição de frequência da dor em pacientes idosas com perda de massa óssea, antes e depois do Programa Fisiomotriz

\begin{tabular}{|c|c|c|c|c|c|}
\hline \multirow[b]{2}{*}{ ID / Situação } & \multicolumn{2}{|c|}{ SAT } & \multicolumn{2}{|c|}{ SDT } & \multirow[b]{2}{*}{ (P) } \\
\hline & f & $\%$ & f & $\%$ & \\
\hline$<1$ (Sem dor) & - & - & 04 & 13,8 & \\
\hline 1 a 2,5 (MB) & 02 & 6,9 & 07 & 24,2 & \\
\hline 2,5 a $5(\mathrm{MO})$ & 08 & 27,6 & 13 & 44,8 & 0,0006 \\
\hline 5 a 7,5 (MA) & 08 & 27,6 & 05 & 17,2 & \\
\hline 7,5 a 10 (SE) & 11 & 37,9 & - & - & \\
\hline Total & \multicolumn{2}{|c|}{29} & \multicolumn{2}{|c|}{29} & \\
\hline
\end{tabular}

Legenda: ID = Índice de Dor; SAT = Situação Antes do Tratamento; SDT = Situação depois do Tratamento. 
A Análise de Variância de Kruskal Wallis utilizada com o teste $\mathrm{Chi}^{2}$ embutido sobre os escores de dor revelou índice de valor $\mathrm{Chi}^{2}=0.0006$, com $1(\mathrm{gl}), \mathrm{p}<0.05$. Este resultado revela que o grupo antes portador de dor severa (grupo 2) apresentou um decréscimo, em dor, ainda maior do que o do grupo 1.

Visualizando-se a Tabela 2, é possível perceber a grande evolução obtida pelas avaliadas com índice de IG > 5.0, após o programa fisiomotriz. Antes da intervenção (SAT), todos os indivíduos do grupo apresentavam padrão de autonomia funcional fraco, já na avaliação pós-intervenção (SDT), a grande maioria distribuiu-se entre fraco, regular, bom ou muito bom. Estatisticamente foi definido, com aplicação do teste Qui ${ }^{2}$ aplicado aos dados do teste de GDLAM, p = 0, 0001, < 0, 05, levando à rejeição da hipótese nula, condição que permite estabelecer uma posição de que as alterações dos padrões iniciais de autonomia funcional (PAF), melhorando para regular, bom e muito bom, foram vinculadas, pelo menos em parte, ao programa fisiomotriz.

Tabela 2 - Distribuição da freqüência do IG em pacientes idosas com perda de massa óssea e dor acima e abaixo de 5.0 na Escala Analógica Visual, Antes e Depois do Programa Fisiomotriz e, sua relação com o Padrão de Autonomia Funcional

\begin{tabular}{|c|c|c|c|c|c|c|c|c|}
\hline \multirow{2}{*}{$\begin{array}{l}\text { PAF / Situação de IG em } \\
\text { SAT e SDT }\end{array}$} & $\mathrm{SAT}>5.0$ & $\mathrm{SDT}>5.0$ & \multirow[b]{2}{*}{ (Sig) } & \multicolumn{2}{|c|}{$\mathrm{SAT}<5.0$} & \multicolumn{2}{|c|}{$\mathrm{SDT}<5.0$} & \multirow[b]{2}{*}{ (Sig) } \\
\hline & f $\quad \%$ & f $\quad \%$ & & f & $\%$ & $\mathrm{f}$ & $\%$ & \\
\hline$>27,42(\mathrm{~F})$ & $\begin{array}{c}14 \\
100\end{array}$ & $\begin{array}{c}02 \\
14,3\end{array}$ & & - & - & - & - & \\
\hline 27,42 a $24,98(\mathrm{R})$ & - & $\begin{array}{c}04 \\
28,6\end{array}$ & & 09 & 60,0 & 01 & 6,7 & \\
\hline 24,98 a 22,66 (B) & - & $\begin{array}{c}06 \\
42,8\end{array}$ & 0,0001 & 04 & 26,7 & 02 & 13,3 & 0,0008 \\
\hline$<22,66(\mathrm{MB})$ & - & $\begin{array}{c}02 \\
14,3\end{array}$ & & 02 & 13,3 & 12 & 80,0 & \\
\hline Total & 14 & 14 & & & 5 & & 00 & \\
\hline
\end{tabular}

Legenda: IG = Índice de GDLAM; PAF = Padrão de Autonomia Funcional $(\mathrm{F}=$ Fraco; $\mathrm{R}=$ Regular; $\mathrm{B}=\mathrm{Bom}$; MB $=$ Muito Bom); SAT $>5.0=$ Situação Antes do Tratamento dos pacientes com perda de massa óssea e dor acima de 5.0; SDT $>5.0=$ Situação depois do Tratamento dos pacientes com perda óssea e dor acima de 5.0. SAT $<5.0=$ Situação Antes do Tratamento dos pacientes com perda de massa óssea e dor abaixo de 5.0; SDT $<5.0=$ Situação depois do Tratamento dos pacientes com perda óssea e dor abaixo de 5.0

Na Tabela 3 os resultados demonstraram percentual de redução médio do Índice GDLAM (IG) em idosas com índice > 5.0, em torno de $21 \%$ entre o antes e o depois do programa fisiomotriz. Estatisticamente foi definido, com aplicação do Teste de Kruskal-Wallis, p < 0,0001, menor que 0, 05, portanto, rejeita-se a hipótese nula e admite-se que o programa fisiomotriz dispensado em mulheres idosas com osteopenia e/ou osteoporose apresentou resultados altamente significativos e associados à diminuição do sintoma dor e ampliação dos movimentos. Ainda nessa tabela são apresentados, referentes ao grupo com índice de dor na escala analógica visual $<5.0$, resultados que demonstram percentual de redução médio do índice de IG, em torno de 16\% depois da intervenção do programa fisiomotriz. Estatisticamente foi definido, com aplicação do Teste de Kruskal-Wallis, p < 0,0001, menor que 0,05, portanto, desta forma, está estatisticamente comprovado que o programa fisiomotriz dispensado em mulheres idosas, com osteopenia e ou osteoporose, apresenta significativa diminuição do sintoma dor e ampliação dos movimentos. 
Tabela 3 - Distribuição média do Índice de GDLAM (IG) em pacientes idosas com perda de massa óssea e dor maior e menor que 5.0 na Escala Analógica Visual, Antes e Depois do programa fisiomotriz

\begin{tabular}{rrrccc}
\hline $\mathbf{N}$ & IG & SAT & SDT & \%R & (P) \\
\hline $\mathbf{1 4}$ & $>\mathbf{5 . 0}$ & 32,09 & 25,19 & 21,12 & $<0,0001$ \\
$\mathbf{N}$ & IG & SAT & SDT & \%R & (p) \\
$\mathbf{1 5}$ & $<\mathbf{5 . 0}$ & 25,07 & 20,99 & 16,24 & $<0,0001$ \\
\hline
\end{tabular}

Legenda: IG = Índice de GDLAM; SAT = Situação Antes do Tratamento; SDT = Situação Depois do Tratamento; \% R = Percentual de Redução do IG em relação à SAT

A Figura 1 apresenta os menores e maiores escores, mediana e quartil, assim como os valores para a frequência na relação respectiva da redução de IG em SAT e SDT, dor acima e abaixo de 5.0, simultaneamente, com $\mathrm{N}=6 ; 42,8 \%$ equivalente a bom e $\mathrm{N}=12 ; 80 \%$ equivalente a muito bom.

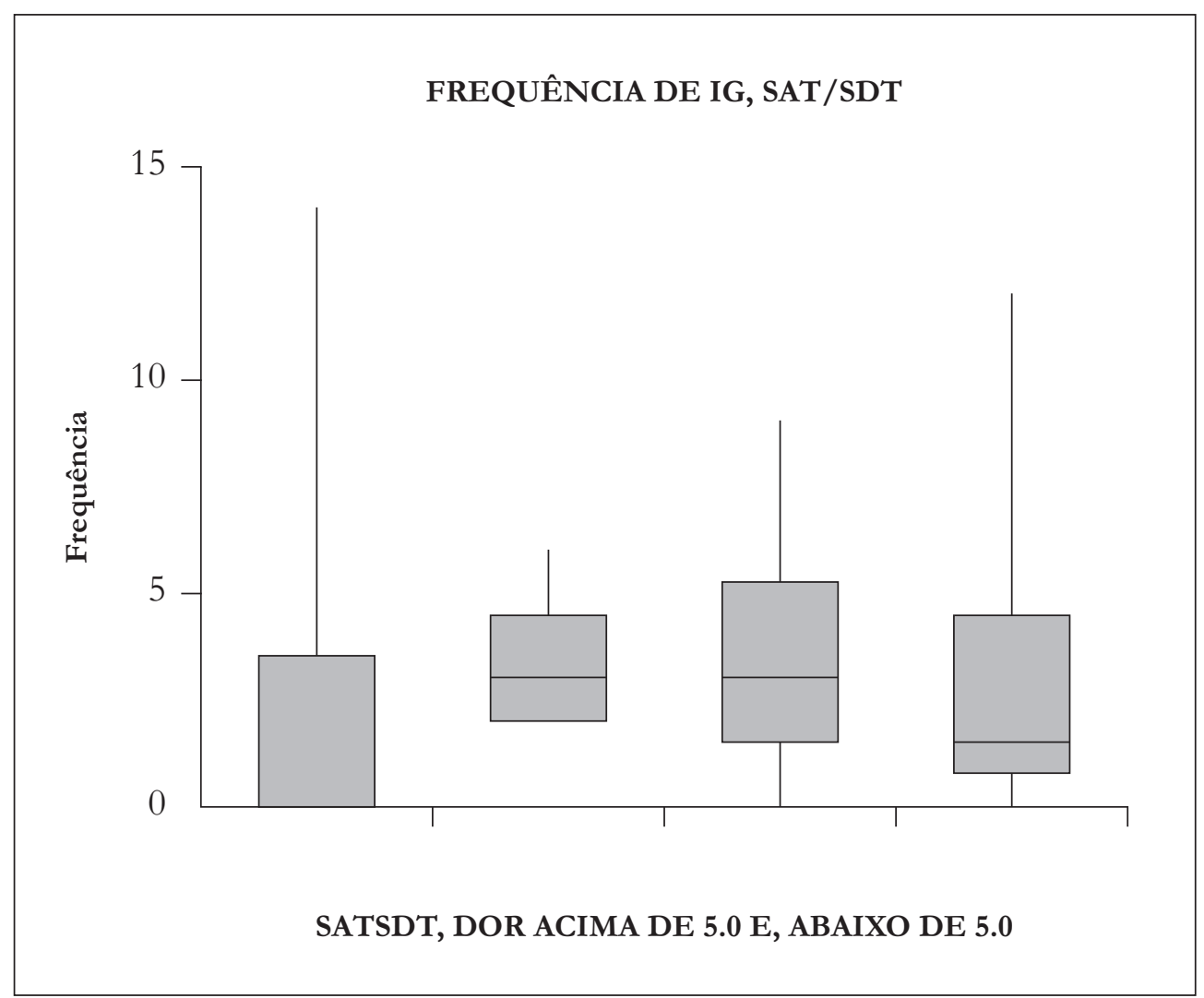

Figura 1 - Apresenta os menores e maiores escores, mediana e quartil, assim como os valores para a freqüência na relação respectiva da redução de IG em SAT e SDT, dor acima e abaixo de 5.0 simultaneamente

Legenda: IG = Índice de GDLAM; SAT= Situação Antes do Tratamento; SDT= Situação Depois do Tratamento

Já a Figura 2 apresenta curva de dispersão com percentual de redução médio de IG, respectivamente, em $21 \%$ e $16 \%$ depois do tratamento cinesioterápico/fisiomotriz, nos grupos dor acima e abaixo de 5,0 na escala analógica, com resultados estatísticos altamente significantes na relação entre a diminuição do sintoma da dor e ampliação dos movimentos. 


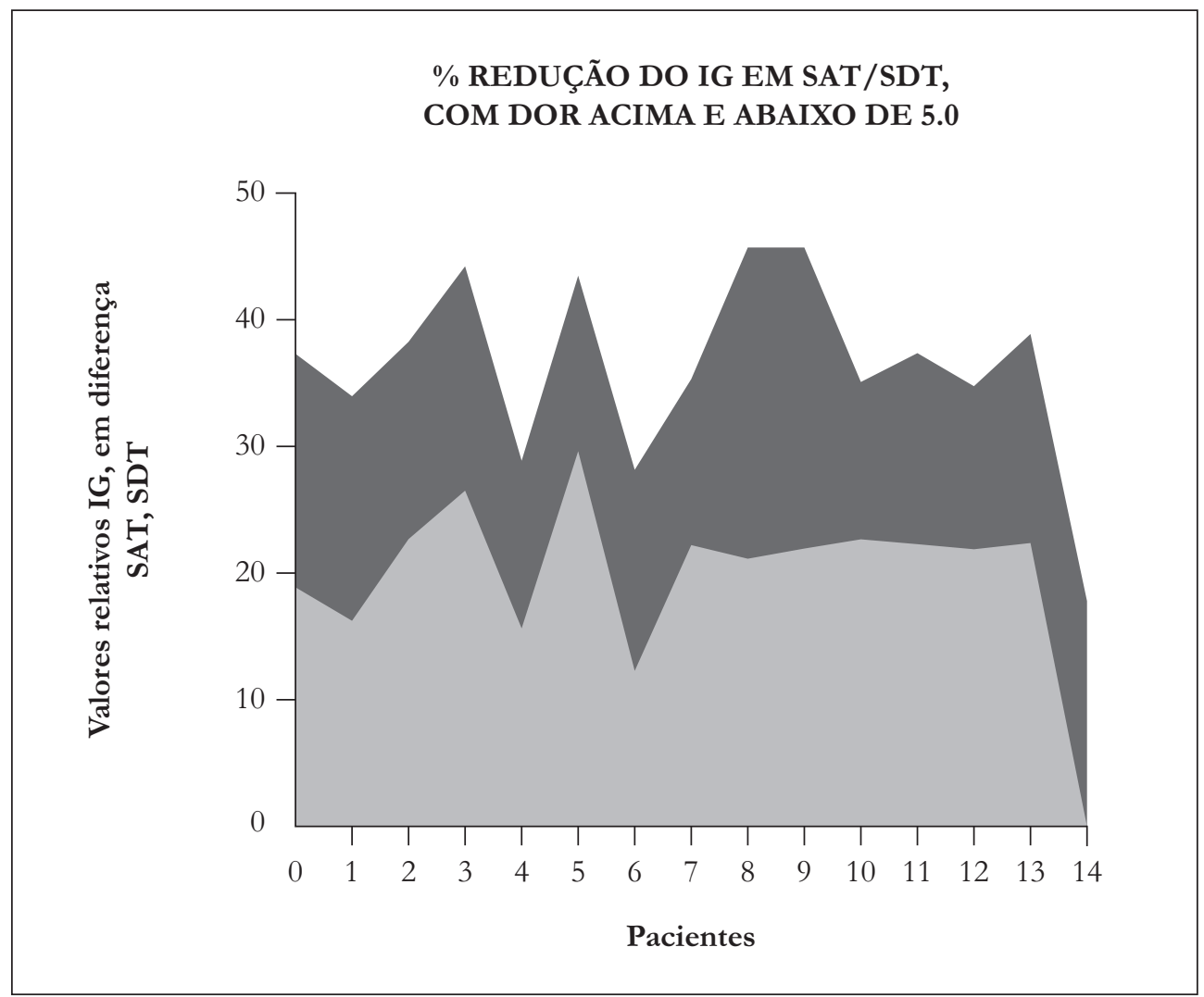

Figura 2 - Curva de dispersão com percentual de redução médio de IG, antes e o depois do tratamento, cinesioterápico/fisiomotriz, nos indivíduos com dor acima e abaixo de 5,0 na escala analógica

Legenda: IG = Índice de GDLAM, SAT= Situação Antes do Tratamento, SDT= Situação depois do Tratamento

\section{Discussão}

A dor crônica é encarada como um fator complexo que escapa ao paradigma biomédico e tem impulsionado mudanças e adaptações no sentido de preencher lacunas e gerar novas possibilidades no cuidado à saúde, principalmente do idoso. O programa fisiomotriz aqui aplicado destinou-se, em sua essência, a contemplar o maior número de itens sugeridos pela Sociedade Brasileira de Medicina do Esporte e da Sociedade Brasileira de Geriatria e Gerontologia (30), principalmente no que diz respeito à socialização e ao trabalho em grupo que determina a terapia física como algo prazeroso e de fácil adesão para o idoso. De certa maneira, a diminuição da dor relatada foi bem abrangente, visto que, conforme descrito na Tabela 1, antes da aplicação do Programa (SAT), nenhuma das idosas apresentou situação de não dor. O maior percentual (independente) delas se enquadrava na condição de dor severa $(37,09 \%)$ e outro grande percentual composto por portadoras de níveis moderados (MO) e moderado elevado (MA), perfazendo um quantitativo de 55\% de todas as idosas. A minimização de dor para todas as participantes se estendeu, especificamente relatando, à condição de não dor pós-programa para quase $14 \%$ das idosas, enquanto que o grupo de dor baixa (MB) aumentou e a condição de dor moderada passou de 27,6 para 44,8. Este último dado, indicando que as idosas que sentiam dor forte passaram, em um grande percentual, para a situação de dor moderada. Assim sendo, o grupo situado, inicialmente, no nível severo de dor passou a um estágio de completa ausência de dor.

Esse resultado, assim como os apresentados nas Tabelas 2 e 3, indicam concordância com o pensamento de Lin et al. (10), que formalizam a ideia de que, com a utilização de exercícios isométricos sob forma livre e contra-resistidos, é possível minimizar quadros de edemas e de outros processos inflamatórios, condições que tendem a favorecer as funções circulatórias do organismo, levando-o à oposição a dor. 
Esses mesmos autores explicam, ainda, que movimentos realizados sob orientação personalizada, auridos a partir de relaxamento e adequados processos respiratórios, podem auxiliar a harmonização de áreas doloridas, sob fluxo de práticas exteroceptivas que comungam para um estado bom de equilíbrio funcional e respiratório como um todo. Essas noções se aplicam ao trabalho de Silva e Lage (11), os quais mostram que exercícios de mentalização, incorporados com posturas estáveis e confortáveis, podem favorecer a inibição de certas vias de dor, ou pontos desta, limitando os canais de dor e, consequentemente, minimizando-a. Em uma linha associada, estudos têm demonstrado os benefícios da exercitação física para indivíduos osteoporóticos, minimizando dosagens medicamentosas e aumentando a mobilidade corporal e massa óssea (12).

Logicamente, a minimização da dor apresentada pelos participantes do estudo teve repercussão sobre o fator da autonomia funcional dos grupos. Acredita-se que o fato de sentir menos dor, ou o alívio proporcionado por estados sem dor, conseguido em função do programa, tenha repercutido sobre estados motivacionais, estimulando a participação dos idosos aos exercícios, e consequente maior aderência ao programa.

\section{Conclusão}

Conclui-se que o programa fisiomotriz aplicado em idosas com osteopenia e/ou osteoporose foi eficaz na melhora de sua autonomia funcional, avaliada pelo instrumento GDLAM. Este benefício, em grande parte, pode estar associado à diminuição de dor relatada por essas participantes. O programa mostrou-se efetivo em prevenir o aumento da dor, chegando inclusive a minimizá-la, resultando na aderência das idosas ao programa. Estes ganhos foram independentes do nível de comprometimento individual apresentado antes do início da intervenção realizada.

Assim, neste estudo ficam evidentes os benefícios que um programa de atividades físicas bem elaborado pode oferecer a idosos com comprometimentos físicos. Definido como fisiomotriz, este programa de aplicação de exercícios cinesioterápicos regulares e adequados às necessidades e possibilidades funcionais de idosas com diferentes níveis de comprometimento físico e dores crônicas pode ser pensado como uma forma eficaz de redução da dor, condição que facilita a aderência e imprescindível ao logro de qualquer tipo de benefício que se possa pensar proporcionável pela atividade física regular. Considerando-se as evidências da literatura em relato a tais benefícios, vale a pena qualquer investimento a ser feito no sentido de se facilitar a entrada e a aderência de indivíduos idosos à sociedade ativa. O caminho via exercício físico pode ser uma promissora alternativa para tanto, como se demonstra nos resultados da presente pesquisa, apontando que o estudo da fisiomotricidade é um campo fértil para o desenvolvimento de técnicas que, assim como o programa fisiomotriz aplicado aqui, busquem algum tipo de ganho em indivíduos de diferentes populações.

\section{Referências}

1. Andrade FA, Pereira LV, Sousa FAEF. Mensuração da dor no idoso: uma revisão. Rev Latino-am Enfermagem. 2007;14(2):37-45.

2. Andrade FA, Pereira LV, Souza FAEF. Mensuração da dor no idoso: uma revisão. Rev Latino-am. Enfermagem. 2006;14(2):271-6.

3. Dellarosa MSG, Pimenta CAM, Matsudo T. Prevalência e caracterização da dor crônica em idosos não institucionalizados. Cad Saúde Pública. 2007;23(5):1151-1160.

4. Almeida HO, Versiani ER, Dias AR, Novaes MRCG, Trindade EMV. Adesão a tratamentos entre idosos. Treatment adherence among the elderly. Com. Ciências Saúde. 2007;18(1):57- 67. 
5. Pimentel CAM. Dor crônica, terapiacognitiva comportamental e o enfermeiro. Rev Psiq Clín. 2001;28(6):288-94.

6. Budó MLD, Resta DG, Nicolini D, Büttenbender E, Pippi MC, Ressel LB. A cultura permeando os sentimentos e as reações frente à dor. Rev Esc Enferm. 2007;41(1):36-43.

7. Figueiró JA. A dor. São Paulo: Publifolha; 2000.

8. Cader SA, Silva EB, Vale R, Bacelar S, Monteiro MD, Dantas E. Efeito do treino dos músculos inspiratórios sobre a pressão inspiratória máxima e autonomia funcional de idosos asilados. Motricidade. 2007;3(1):279-88.

9. Caromano C, Ide MR, Kerbauy RR. Manutenção na prática de exercícios por idosos. Revista do Departamento de Psicologia - UFF. 2006;18(2):177-92.

10. Lin TY, Kaziyama HHS, Teixeira MJ. Síndrome dolorosa miofascial. In: Teixeira MJ, Figueiró JAB. Dor. São Paulo: Moreira Jr.; 2001. p. 131-40.

11. Silva GD, Lage LV. Ioga e fibromialgia. Rev Bras Reumatol. [online]. 2006 [acesso 17 nov. 2008];46(1):3739. Disponível em: http://www.scielo.br/pdf/rbv/v46n1/29385.pdf

12. Navega MT, Oishi J. Comparação da qualidade de vida relacionada à saúde entre mulheres na pós-Menopausa praticantes de atividade física com e sem osteoporose. Rev Bras Reumatol. [online]. 2007 [acesso 4 nov. 2008];47(4). Disponível em: http://www.scielo.br/pdf/rbv/v47n4/a04v47n4.pdf

13. Matsudo SM, Matsudo VKR, Barros NTL. The impact of aging on anthropometric, neuromotor, and metabolic variables of physical fitness. Rev Bras Cien e Mov. 2000;8(4):21-32.

14. Cunha CEW, Pontes FLJ, Bacurau RFP, Navarro F. Os exercícios resistidos e a osteoporose em idosos. Rev Bras Prescrição e Fisiologia do Exercício. 2007;1(1):18-28.

15. Geraldes AAR. Efeitos do treinamento contra resistência sobre a força muscular e o desempenho de habilidades funcionais selecionadas em mulheres [dissertação]. Rio de Janeiro: Universidade Castelo Branco; 2000.

16. Amorim FS. Efeitos do treinamento da capacidade aeróbica sobre a qualidade de vida e autonomia de idosos. [dissertação]. Rio de Janeiro: Universidade Castelo Branco; 2002.

17. Aragão JCB. Efeitos da resistência muscular localizada visando à autonomia e a qualidade de vida de idosos. [dissertação]. Rio de Janeiro: Universidade Castelo Branco; 2002.

18. Baptista MR. A prática do yoga sobre a autonomia funcional e qualidade de vida em mulheres senescentes. [dissertação]. Rio de Janeiro: Universidade Castelo Branco; 2004.

19. Vale RGS, Novaes JS, Dantas EHM. Efeitos do treinamento de força e de flexibilidade sobre a autonomia de mulheres senescentes. Rev Bras Ci e Mov. 2005;13(2):33-40.

20. Vale RGS. Avaliação da autonomia funcional do idoso. Fitness \& Performance Journal. 2005;4(1):13-7.

21. Vale RGS, Barreto ACG, Novaes JS, Dantas EHM. Efeitos do treinamento resistido na força máxima, na flexibilidade e na autonomia funcional de mulheres idosas. Rev Bras Cineantropom Desempenho Hum. 2006;8(4):52-8.

22. Kurita GP, Pimenta CAM. Adesão ao tratamento da dor crônica: estudo das variáveis demograficas, terapeuticas e psicossociais. Arq Neuropsiquiatria. 2003;61(2-b):416-25.

23. D’Alencar BP, Mendes MMR, Jorge MSB, Guimarães JMX. Biodança como processo de renovação existencial do idoso. Rev Bras Enferm. [online]. 2008 [acesso 4 nov. 2008];61(5). Disponível em: http:/ / www.scielo.br/scielo.php?script=sci_arttext\&pid $=$ S003471672008000500013\&lng $=$ en\&nrm $=$ iso 
24. Melzack R, Wall P. The challenge of pain. New York: Basic Books; 1983.

25. Soares, CVBC Verificação da eficácia de um método fisiomotriz sobre a dor e autonomia funcional de idosas com perdas de massa óssea. 2008. [dissertação]. Rio de Janeiro: Universidade Castelo Branco; 2008.

26. Soares KVBC, Silva Vernon F. Fisiomotricidade em dor, autonomia funcional e massa óssea de idosas osteoporóticas. Motriz. 2009;15(2):273-283.

27. Soares KVBC, Neto NTA, Silva Vernon F. Fisiomotricity of adequate intensity the pain thresholds:effectiveness on the pimax and pemax of aged women with osteoporosis. FIEP Bulletin. 2009;79(Special II):497-500.

28. Castro KVB, Silva ALS, Silva Vernon F. Fisiomotricidade de intensidade adequada a limiares de dor: eficácia sobre o ganho de massa óssea de idosas osteoporóticas. Fisioter Bras. 2008;9:315-21.

29. Queiros VRL, Soares KVBC, Silva Vernon F. Estudo comparativo sobre a autonomia funcional de idosas praticantes de fisiomotricidade e idosas praticantes de atividade física regular. In: CONGRESSO EUROAMERICANO DE MOTRICIDADE HUMANA, 3., Murcia. Anais... Murcia, Espanha: Murcia; 2009.

30. Nóbrega ACL, Freitas EV, Oliveira MAB, Leitão MB, Lazzoli JK, Nahas RM, et al. Posicionamento oficial da Sociedade Brasileira de Medicina do Esporte e da Sociedade Brasileira de Geriatria e Gerontologia: atividade física e saúde no idoso. Rev Bras Med Esporte. 1999;5(6):207-11.

Recebido: 26/01/2009

Received: 01/26/2009

Aprovado: 10/11/2009

Approved: 11/10/2009 